\title{
Control Architecture Scalable Dedicated to Braille Panels for Displaying Information on Public Places
}

\author{
Roque, Alexandre S. \\ Department of Engineering \\ and Computer Science \\ Center for Assistive \\ Technology - CTA \\ Regional Integrated University \\ of High Uruguay and Missions \\ Santo Ângelo, RS, Brazil
}

\author{
Silva, Denílson R. \\ Department of Engineering \\ and Computer Science \\ Center for Assistive \\ Technology - CTA \\ Regional Integrated University \\ of High Uruguay and Missions \\ Santo Ângelo, RS, Brazil
}

\author{
Santos, Cristina P. \\ Department of Engineering \\ and Computer Science \\ Center for Assistive \\ Technology - CTA \\ Regional Integrated University \\ of High Uruguay and Missions \\ Santo Ângelo, RS, Brazil
}

\begin{abstract}
This paper proposes a control architecture for driving a panel Braille, that works as an aid in the reading of information by visually impaired people and also for applications for teaching Braille. To validate a prototype it was developed containing the control algorithm, which uses two techniques together: the conversion of characters in dot matrix in software and hardware multiplexing signals, and both techniques are scalable to jointly control the display of Braille characters on the panel. The panel allows the rewriting and updating size to provide new operating parameters.
\end{abstract}

\section{General Terms}

Embedded systems, Assistive technology, Control Architecture

\section{Keywords}

Demultiplexing signals, Solenoid actuators, Braille panel

\section{INTRODUCTION}

The new Information and Communication Technologies (ICTs) have become, increasingly, important instruments of culture, and can be used as Assistive Technology (AT) [1], [2]. The TA includes all the features and services that contribute to provide or expand functional abilities of people with disabilities and, consequently, promote independent living and inclusion [3]. Among these technologies stand out those for people with visual disabilities covering areas such as, for example, moving in different environments [4], identification of items in appropriate [5], reading and printing of documents [6], [7], [8], [9].

Considering the different scenarios that drive research focused on the development of Assistive Technologies is emphasized in this paper, those in which information is made available in text format, expressed through posters, posters, cards and other gifts at bus stops, airports, bus stations and universities, which have information centers (electronic billboards) constantly being updated. This scenario is a daily reality in the lives of the visually impaired and causes many difficulties of access to information to such individuals.

In this perspective, the embedded systems emerge as an alternative to the Assistive Technology area as may be directed to the development of solutions that incorporate adequate resources to resolve the problems caused by the described scenario. As an example of its use it can be mentioned the Braille Display, also known as "Braille Lines" that allows the printing of electronic texts dynamically in real time promoting great contributions under the assistive technologies [4], [6], [8]. However, despite the benefits of using this technology there are limitations including: (a) the specific size for reading digital text in real time; (b) high cost and (c) does not have the scalability and flexibility features.

Thus, the demand for new technological solutions that benefit people with visual disabilities in access to information and the limitations of existing resources has fueled the development of this study, which has the intention to provide a configurable control technique to compose an Embedded System, capable of receiving digital texts through a server, storing it and translating the text to Braille system. This is intended to promote the subsidies needed for the design of Braille panels that are expandable in relation to its size, allow the provision of electronic texts dynamically and in real time and that are developed with low-cost technologies.

The proposed control technique emphasizes the use of demultiplexers, which are aligned so as to compose a drive matrix with row and column decoding, similar to the form used in memory subsystems. The use since component in conjunction with the control algorithm which converts the Braille characters in a dot matrix, enables control braille panels of a wide variety of sizes.

This paper is organized into five sections: Section II discusses the topics Vision, Braille, Assistive Technologies, Solenoids and Embedded Systems related to the proposal of context; Section III describes the development of the work with the programmed set of operating instructions and prototyped hardware for technical tests; finalizing the IV section contains the conclusions and future prospects.

\section{THEORETICAL BACKGROUND 2.1 Vision}

Visual impairment is a sensory limitation that can reach different levels, being able to annul the capacity to see. For the visually impaired, performing simple daily tasks, entails limitations or impairments in social interaction. The acquisition of concepts, direct access to the written word, orientation and independent mobility are examples of activities that have a considerable challenge for the visually impaired. Because of its scope and complexity there are several definitions and classifications of visual impairment, discussed in several countries, emphasized by the World Health Organization [1].

In Brazil, the decree no. 5296 of $12 / 02 / 2004$, which consolidated the Federal Laws numbers 10.048 and 10.098 dealing with the accessibility of the disabled person and person with reduced mobility. For greater accessibility for the 
visually impaired, there is a system that can make a person with visual impairment have a more autonomous life, this system is known as Braille.

\subsection{Braille Language}

Braille is a tactile language for reading texts, used for display. It consists of 63 signals obtained by combining six points (in relief) that are grouped into two vertical and juxtaposed rows of three points each [2], as it can be seen in "Fig. 1" (a). In the cell braille points are numbered 1 through 6 and Braille code results from a combination of them, "Fig. 1" (b). This cell does not exceed the tactile field and can be identified quickly because of its shape it fits exactly into the pulp of the finger [2].

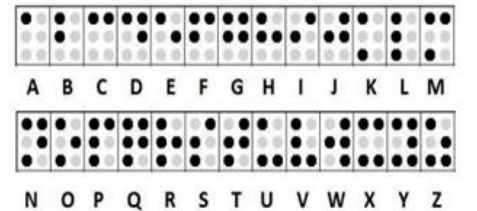

(a)

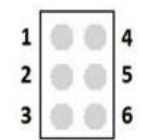

Fig. 1: (a) Braille Alphabet. (b) Braille Cell.

The dimensions of a Braille cell vary according to country and may have different values for the same territory region [9].

\subsection{Braille Language}

Assistive technology is an area of knowledge, interdisciplinary feature, which includes products, resources and services to facilitate the development of activities of daily living for people with disabilities, aiming their autonomy, independence, quality of life and social inclusion.

The braille lines (also known as Braille or Braille Electronic screen) are devices connected to the serial port or USB (Universal Serial Bus) of a computer and presenting digital text in a row of Braille cells formed by groups of pins. The configuration of these pins changes as the user navigates through text [10]. A "Fig. 2" displays examples of product lines Braille market.

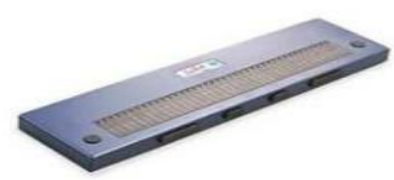

(a)

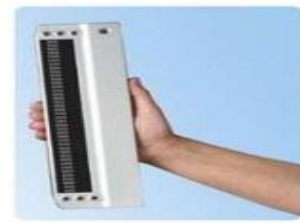

(b)
Fig. 2: (a) Braille line Keika. (b) Braille Line SuperVario40.

\subsection{Actuators}

For the display of Braille characters on the high reveal that can be actuated electronically, the solenoid is commonly used. The following are examples of solenoids are shown in "Fig. 3".

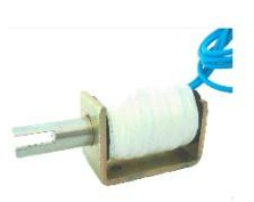

(a)

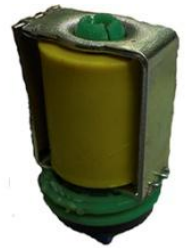

(b)

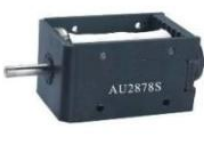

(c)
Fig. 3: (a) Commercial use (b) Used in water pumps. (c) International model.
The solenoid is actuated electromechanically by a coil. When energizing the coil is produced (induced) a magnetic field, which works in a similar way as a natural magnet, repelling the metal pin contained within, elevating it to the device surface. This provides the necessary relief for forming the braille reading of the cell [11].

Commercial solenoid actuators "Fig. 3 a)" has an average cost of $\$ 10$ per unit, since the reused an electronic tap "Fig. 3 b)", has a cost of $\$ 4$ per unit, the solenoid imported from China has a cost of $\$ 5.00$ US dollars per unit, considering imports into Brazil.

In the same segment others works related information display Braille texts, for example [6] and [7]. In the present study for control system validation, also were used solenoids actuators in conjunction with LED lights, although not exclusive use of them, as is highlighted in the approach proposed control architecture.

\subsection{Embedded Systems}

An embedded system or embedded system is characterized by being a system having some kind of processing carried out by microprocessors which execute repeatedly a control software (internal control algorithm) controlling the embedded system and its peripherals [12], [13] is also defined by the IEEE [14] standard as "a computer system that is part of a larger system and implements some of the requirements for this system." This definition, established for more than two decades, is still valid, although from the past few years, some authors have driven it to complement it. Steve Heath defines it as "one based on a microprocessor system, which is designed to control a function or a range of functions, and not to be programmed by the end user as with computers" [15].

Researches like the computer vision-based object recognition [16], a light news reader for the visually impaired person [6], Braille lines and the Braille printer [8] are examples of devices that are part of this concept. This work is characterized as an Embedded System because it meets some characteristics, such as having a microcontroller that controls specific functions, have fewer components compared to a computer as highlighted [17].

\subsubsection{Microcontrollers}

The work of Rana et al [16] presents design and implement a cost effective and yet flexible and powerful home security system using the GSM technology that uses ATMEGA328P microcontroller in control system, since the work Gartseev [18] uses the same design for a low cost of a platform for learning robotics, work of Cardesin et al [19] with presents a low-cost 18W intelligent LED driver for energy savings. Based on these works that demonstrate the widespread use of this microcontroller, it was also used for the control architecture tests.

Today there is a variety of microcontrollers, with several possibilities for implementation from simple designs to very complex applications. The microcontroller ATmega328P from Atmel AVR family of 28-pin, presents characteristics such as Harvard architecture, ie, data memory and program memory separated. It also has an operating frequency of around $16 \mathrm{MHz}$. As this frequency is not divided, as in the 8051 microcontroller and some PICs where the frequency divided by 12 and 4, respectively, it becomes faster than the other microcontrollers.

Instructions in the program memory are executed with a single level pipelining. While an instruction is being executed, 
the next instruction is pre-obtained from the program memory. This concept enables instructions to be executed in every clock cycle, and gets around by 1MIPS MHZ. All these features prove the existing concern regarding the performance when using this type of microcontroller [20].

Microcontrollers have flash memory (used to store firmware), SRAM (volatile storage memory that loses data when interrupting the supply of power) and EEPROM (capable of storing information even if the power interruption).

The EEPROM memory is used for storage of data and making this device need not always connected to a server. Each of the 14 digital pins on the ATmega328P can be used as input or output. Each pin can provide or receive a maximum of $40 \mathrm{~mA}$ and operates 5 volts.

\subsubsection{EEPROM Memory}

Parallel EEPROM allows data to be stored and updated byte by byte, or complete sector, providing design flexibility. Parallel interface devices offer a high reliability and data retention programming faster than reading the serial interface protocols. Atmel company provides a complete selection of densities (64 kbits to 4 Mbits), operating voltages, packages and device [21].

For the storage of information sent to the microcontroller the EEPROM memory is used which consists of $1 \mathrm{~KB}$ of internal storage, this memory permits storage of ASCII characters. For the use of the EEPROM memory, there EEPROM library which is included in the firmware. For upgrading your memory, communication is required with any server.

\subsubsection{Communication}

The ATMEGA328P allows serial communication in standard TTL UART $(5 \mathrm{~V})$, which is available on digital pins 0 (RX) and 1 (TX). A FTDI USB-TTL converter forwards this serial communication over USB and the FTDI drivers (included in software) provide a virtual port to the computer.

Importantly, the ability to use Pulse Width Modulation (PWM), as it allows to obtain an analog voltage from a digital signal, ie a signal that can take the logic $0(0 \mathrm{~V})$ or $1(5 \mathrm{~V})$ [19].

To the microcontroller be capable of controlling a large number of actuators, a good choice is the use of demultiplexers.

\subsubsection{Recording}

The control algorithm needs to be sent to the microcontroller ATMEGA328P, where you need a software to program it and record it. The AVR manufacturer offers a free program to program microcontrollers, called AVR Studio, in which programs can be developed in assembly language or $\mathrm{C}$ language, using the WinAVR (compiler free C).

For component recording, employs a commercial recorder. Recording can be made directly by the AVR Studio with the appropriate hardware attached to the computer recording. A FTDI USB-TTL converter directs the algorithm via serial communications.

\subsubsection{Multiplexers and Demultiplexers}

The demultiplexers have the function to expand the signal switching capacity, for example used for implementation of virtual channels in networks on chip, uses these digital components for filtering or separate the signal among multiple desired in an optical fiber, makes use of multiplexers to be able to multiplex broadcast radio signals, and others applications [21] [22].

For example, work with demutiplexadores from 4 to 16 lines using a TTL circuit to decode four entries in binary code to a mutually exclusive sixteen output when both inputs G1 and G2 are at a logic low. The demultiplexing function is performed using the four input lines to address the address output line, passing data from one input to another input low logic level (LOW). When any input this high (HIGH), all outputs are HIGH. These demultiplexers are ideal for implementing high-performance memory decoders. All entries are loaded trigger diodes and are provided to minimize defects in the transmission line and thereby simplify the system signal. This work is the use of demultiplexers to expand the number of signals transmitted by the microcontroller in the drive multiple actuators braille panels.

\section{DEVELOPMENT}

\subsection{Methodological Aspects}

For the development of this proposal two aspects were considered: (a) conversion of braille characters dot matrix software, and (b) multiplexing hardware signals. Work interactively and in real time with the technique that transforms a text to be displayed by the panel is possible in embedded systems, due to the use of general purpose microcontrollers, as the families PIC and ATMEL, often adopted for its low cost, ease of finding the Integrated Circuit (IC) market and its efficiency, the technique is programmed and embedded it.

As the IC chosen has a limited number of ports, the DM74LS154 demultiplexer was added, thereby to effect its control is used to multiplexing technique signals, as can be seen in "Fig. 4", where several studies [21], [22], [23] reinforce the common use of this technique.

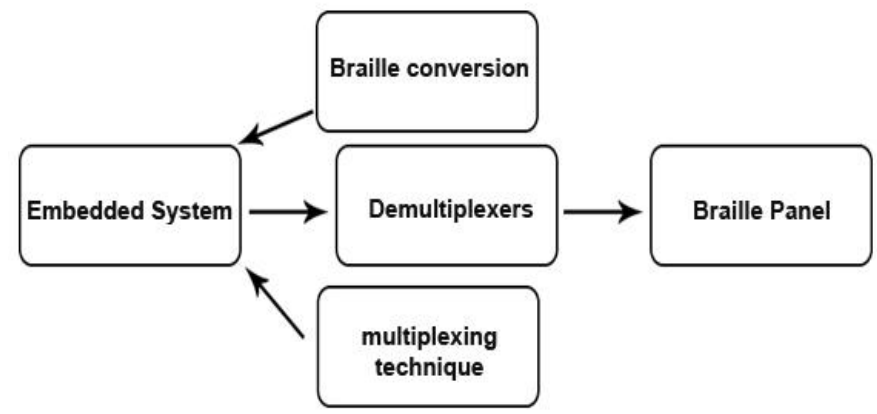

Fig. 4: Representation of Control Technique applied to the system.

Together, the embedded system is implemented multiplexing technique signals, which deals with the activation of demultiplexers, and the conversion of a Braille character, which is responsible for the proper activation of cells, these controls are responsible for the outputs of the Braille Panel.

\subsection{Control System}

To achieve the goals of the work developed a control algorithm capable of receiving a text, which is then converted to an intermediate language (for the purpose of being interpreted by the conversion software for the Braille system) because the microcontroller stores data belonging the ASCII table. Using the text stored converted you can display Braille characters. The system consists of three main parts: 
1. Server: responsible for defining and transmitting the operating parameters of the panel, as its size in rows and columns, and the text to be displayed;

2. Control system/Embedded System: responsible for receiving the server settings and convert the text, using the techniques proposed in the methodology;

3. Panel Hardware: device that can be fitted to various actuators (e.g., solenoids), for the purpose of validating control and in high relief representing a Braille character;

For better understanding of the control algorithm developed, the flowchart in "Fig. 5" shows a more detailed manner the operation.

The control algorithm developed in its startup phase defines all operating parameters entered by the user and starts serial communications. For data after a power failure recovery purposes, a routine data persistence was implemented, which retrieves the latest information stored in the EEPROM memory, for viewing the latest information displayed on the panel.

At the moment there is a reading via serial communication, the received configuration parameters are stored in the device EEPROM.

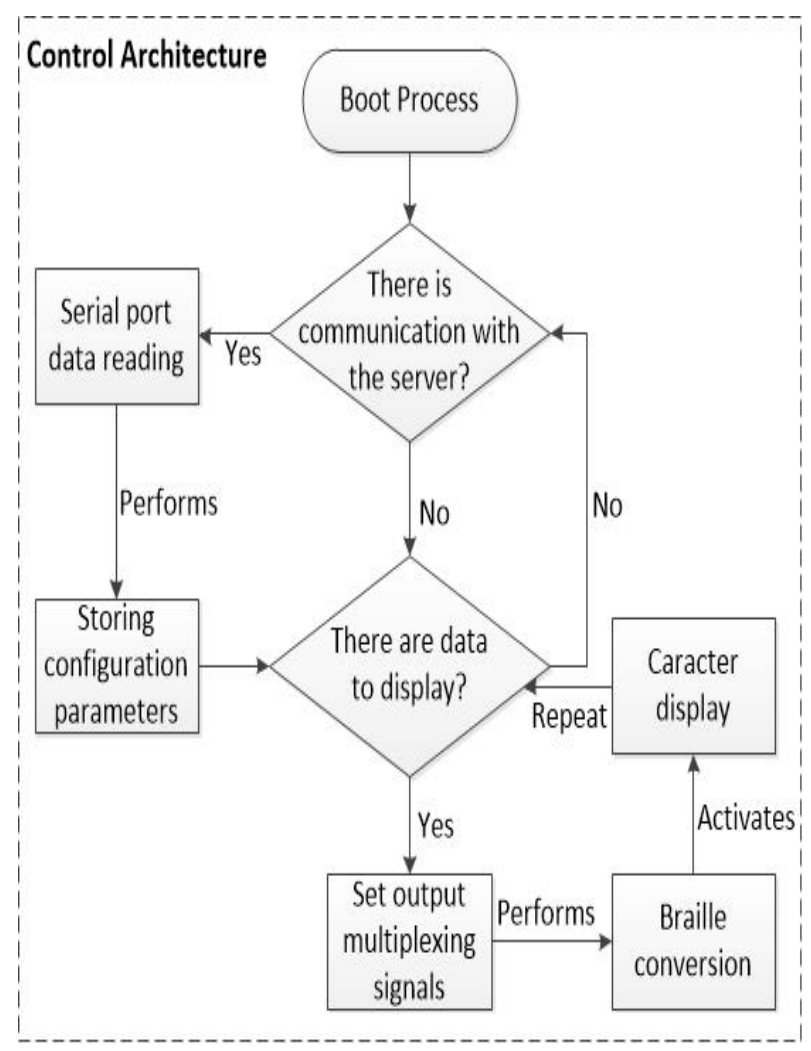

Fig. 5: Flowchart of the proposed control architecture.

To use the EEPROM memory, was divided into two functions "save EEPROM" and "recover EEPROM", "save EEPROM" stores the text received by the "Reading Serial" in the microcontroller memory; "Recover EEPROM" - draws from the text memory stored to be used in "Print". The EEPROM has 1024 bytes of storage, six-byte header, two to enter the number of rows and columns and four for the amount of data to be displayed, the remainder can store the information as displayed in "Fig. 6".
As the largest integer in a byte is 255 , was conducted a calculation to be possible the storage control. To perform this operation was developed the function "EEPROMSave", described below.

void EEPROMSave (String_aSalve, int qLines, int qCols); This function takes three parameters the text to be displayed by the device, the number of lines and the amount of columns, which is the hardware specification. For storing a 16-bit to 8bit integer, the functions are used "highByte" and "lowByte" available for the AVR Studio compiler for Atmel microcontrollers, they divide the whole into two bytes, these are stored in two positions EEPROM memory, thus obtaining integer store 512 .

In the storage of rows and columns their value is converted to two bytes and saved in memory for the text size is checked if it exceeds the size of 512 characters, but not more than is stored only in the first two blocks and the other two is reset, if it exceeds the maximum is stored in the first two and the rest in the other two. At the end of the text is stored as the eighth memory position to a maximum position 1024 , or storing a maximum text characters 1018 and having a limit of 512 rows and columns.

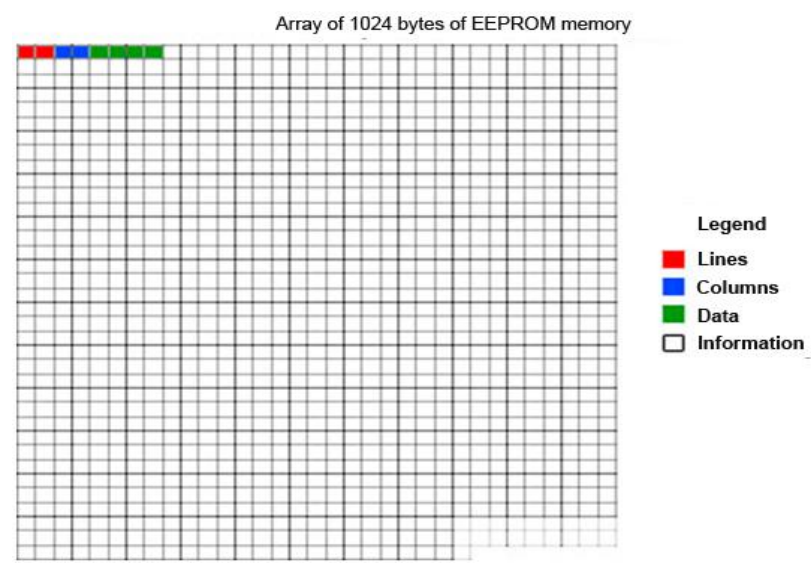

Fig. 6: EEPROM memory usage representation.

To obtain the stored data is used the function "EEPROMRecovering," described below.

string EEPROMRecovering ( int* qtdLines, int qtdCol);

This function retrieves three items of information, the number of lines, the number of columns and the text to be displayed. For the recovery of the divided whole, it is necessary to use the function "word", which takes the "highByte" and the "lowByte", uniting them, thus returning to its original state and getting full value. To recover the text, is recovered the amount stored and added eight value to offset the start at eight in the memory location.

If there is data to display, are used two techniques, one for the letter of the position control, covering the exits and the other to display the Braille character, displaying the corresponding character.

Multiplexing signals is carried out as follows: a function that gets two outputs controls the parameters set for the doors and the demultiplexer that is printing position corresponding to the text. Then, a signal is sent to the "reset" of the demultiplexer, which aims to restart it, then is used torque handling logic to determine which port should be thrown. This logical processing is detailed in "Fig. 7". 


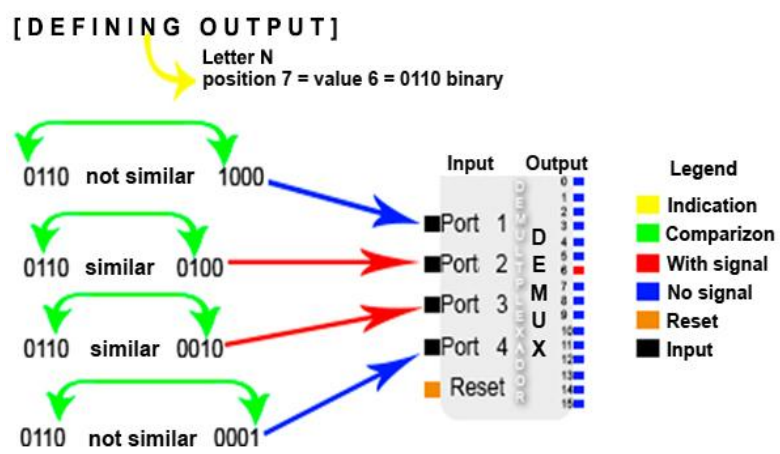

Fig. 7: Selection logic of the output port of the demultiplexer.

In "Fig. 7" above, is used to illustrate the text "defining output" using the seventh letter, "n", which is the sixth element of the array, thus representing six binary "0110", the similarity is analyzed, a comparison bit by bit, like when it will send a signal, otherwise it is not sent, thus triggering the seventh output of the demultiplexer.

The Braille character printing code receives a character and sends a signal to the actuator, depending on the character of the correspondence and the SB, as can be analyzed in Table 1 .

Table 1 - Association of characters in Braille System

\begin{tabular}{|c|c|c|}
\hline Number & Point Activated & Corresponding Character \\
\hline 1 & \begin{tabular}{|l|}
0 \\
0 \\
00 \\
00
\end{tabular} & $\begin{array}{l}a, b, c, d, e, f, g, h, k, 1, m, n, o, \\
\text { p, q, r, u, v, x, y, z, 1, 2, 3, 4, 5, } \\
6,7,8 .\end{array}$ \\
\hline 2 & \begin{tabular}{|l|}
00 \\
00 \\
00
\end{tabular} & $\begin{array}{l}\mathrm{b}, \mathrm{f}, \mathrm{g}, \mathrm{h}, \mathrm{i}, \mathrm{j}, 1, \mathrm{p}, \mathrm{q}, \mathrm{r}, \mathrm{s}, \mathrm{t}, \mathrm{v}, \mathrm{w}, \\
2,6,7,8,9,0 .\end{array}$ \\
\hline 3 & \begin{tabular}{|l|}
00 \\
00 \\
00 \\
0
\end{tabular} & $\begin{array}{l}\mathrm{k}, \mathrm{l}, \mathrm{m}, \mathrm{n}, \mathrm{o}, \mathrm{p}, \mathrm{q}, \mathrm{r}, \mathrm{s}, \mathrm{t}, \mathrm{u}, \mathrm{v}, \mathrm{x}, \\
\mathrm{y}, \mathrm{z} .\end{array}$ \\
\hline 4 & \begin{tabular}{|l|l|}
0 & 0 \\
0 & 0 \\
0 & 0
\end{tabular} & $\begin{array}{l}\mathrm{c}, \mathrm{d}, \mathrm{f}, \mathrm{g}, \mathrm{i}, \mathrm{j}, \mathrm{m}, \mathrm{n}, \mathrm{p}, \mathrm{q}, \mathrm{s}, \mathrm{t}, \mathrm{w}, \\
\mathrm{x}, \mathrm{y}, 3,4,6,7,9,0 .\end{array}$ \\
\hline 5 & \begin{tabular}{|l|}
00 \\
08 \\
00
\end{tabular} & $\begin{array}{l}\mathrm{d}, \mathrm{e}, \mathrm{g}, \mathrm{h}, \mathrm{j}, \mathrm{n}, \mathrm{o}, \mathrm{q}, \mathrm{r}, \mathrm{t}, \mathrm{w}, \mathrm{y}, \mathrm{z}, \\
4,5,7,8,0\end{array}$ \\
\hline 6 & \begin{tabular}{|l|}
0 \\
0 \\
$\circ$ \\
0
\end{tabular} & $\mathrm{u}, \mathrm{v}, \mathrm{w}, \mathrm{x}, \mathrm{y}, \mathrm{z}$ \\
\hline
\end{tabular}

For each point, there are a number of characters that need the respective actuator, for example, the word "labcd", "L" uses Point $1+$ Point 2 + Point3; "A" uses only Point1; "B" uses Point1 + Point2; "C" uses Point1 + Point4; and "D" uses Point $1+$ Point 4 + Point5, as can be visualized in "Fig. 8".

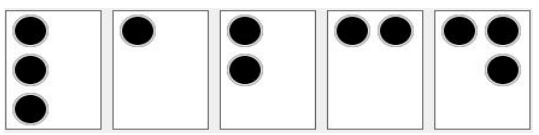

Fig. 8: Demonstration of Braille System with the word ("labcd").
Since only the letter conversion Braille character would not be given the Braille system, so it was developed an intermediate language that translates to the Braille system and must be interpreted by the printing function. To perform the conversion of text to function "translates" receives a given type "string" and checks all character types, for each it is a specific action:

1. Lower case: just add the output;

2. Number: Adds the ' $\mathrm{N}$ ' letter to number indication;

3. Uppercase letter: add the letter ' $L$ ' to uppercase indication;

4. Uppercase letters in sequence: adds the 'LL' letters to indicate an uppercase word.

Thus, making the conversion software for the Braille system meet all the alphabet and make the visually impaired can read all the information without losing any text.

To receive data, the microcontroller has a USB serial communication, these data need to contain a specific header, number of panel rows and columns to be used, followed by the information that can be represented as follows:

[Number of rows, number of columns] data to display

Example:

[1,16] defining output

For updating the hardware, software was developed in C \#, demonstrated in "Fig. 9", connected from the computer's USB port, and you can predefine the panel size, the message to be transmitted and a preview of how it will be displayed.

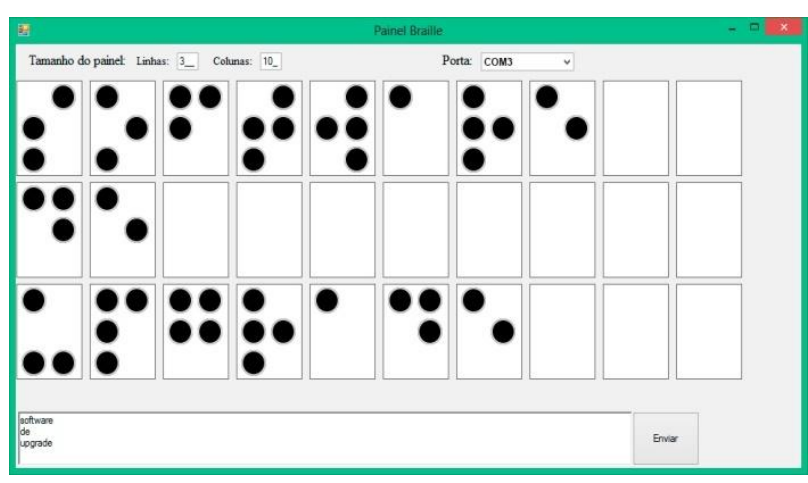

Fig. 9: Display screen of the Braille Panel Update Software.

For software configuration must be set "Lines" and "Columns", corresponding to the dimensions of the panel, as these data are sent in the form header, if not correctly, there will be problems displaying information. A list of available communication port, where should know what port to be used for sending the update appears. In the text field, all typed text is automatically converted to the Braille system and displayed a preview of how it should appear on the panel.

This software was developed for sending control data embedded system.

\subsection{Control Hardware}

To complement control architecture is proposed a form of links of hardware elements. One ATMEGA328P microcontroller that is connected to a server, the demultiplexers DM74LS154 and solenoid or other actuator 
suggested by a designer was used. The following "Fig. 10" illustrates the activation pins that represent the characters.

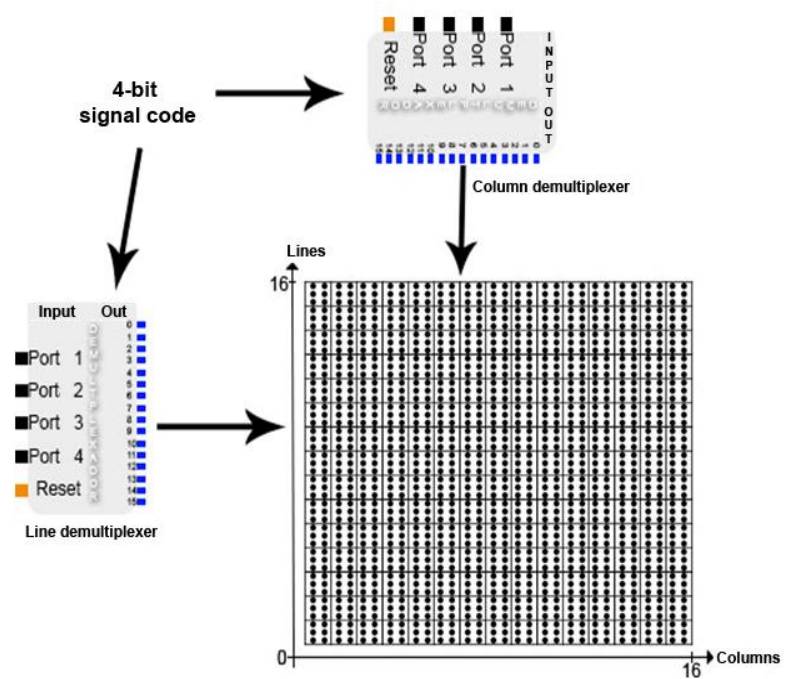

Fig. 10: Representation of the connections to actuation pins of the characters.

The control algorithm allows actuation of the outputs. Thus, when the driver is performing the "impression" of the Braille character, is thrown only one character per output of the demultiplexers. For individual firing of characters, one of which demultiplexers control line must be activated and the other controlled the output column, as can be seen in "Fig. $10 "$.

A demultiplexer controls the actuation lines of the panel, each output is connected to the "reset" each demultiplexer "Fig. 11" thus prevents some other to fire incorrectly, when the output is triggered for one of the lines, so you can use the desired demultiplexer.

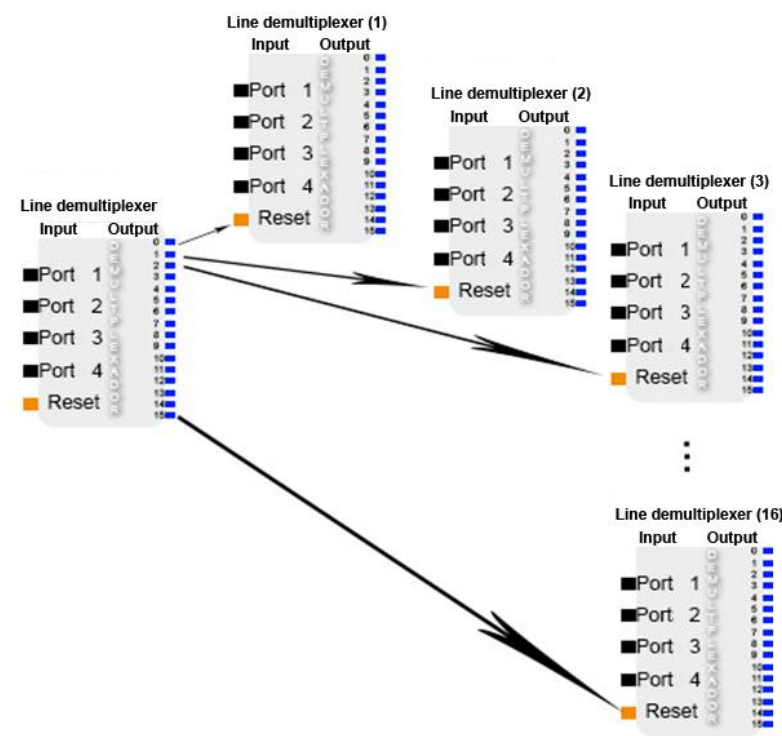

Fig. 11: Actuation connections between multiplexers.

The example illustrates the need for a demultiplexer for each row of a project and a demultiplexer for each 16 columns of character, may be cited the example of "braille", which has 32 characters per one line, in this case requiring 2 (two) demultiplexers for columns and 1 (one) for line.
The outputs of all characters are connected, in which the determination of which characters to be activated depends on the algorithm set by the demultiplexers. The line demultiplexer releases the actuation of the column demultiplexers, and invoking one of the Braille character, as detailed in "Fig. 12".

The example of "Fig. 12", a signal is sent to the demultiplexer lines that informs to trigger the first position of the demultiplexer lines, as was also sent a signal to trigger the seventh column of the demultiplexer columns, therefore, results in the activation of the Braille cell in position $1 \times 7$ of Braille Panel.

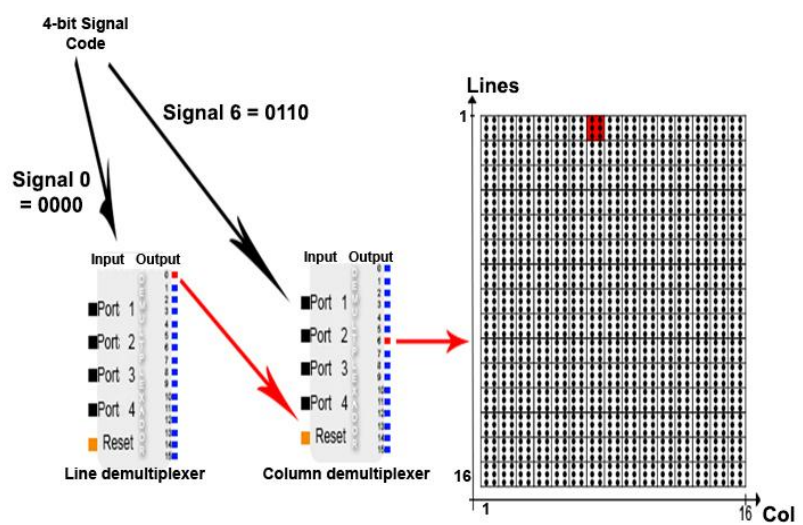

Fig. 12: Activation the Braille Cell (1x7).

The Braille character can be composed of six solenoids, where each has a ground wire and the power to the drive, the "fig. 13 " illustrates this form of braille cell.

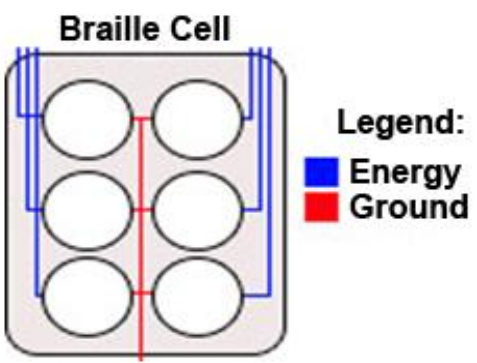

Fig. 13: Connections of Cell Braille.

For the activation of the solenoid character is necessary to switch the points to be informed whether or not fire, along with the column and row, which is the position in which part of the panel will be displayed, can be interpreted by the following equation. $\begin{aligned} & \text { Letter } \\ & \text { Point } 3+\text { Point } 4+\text { Point } 5+\text { Point6 }\end{aligned} \underset{\text { Column }}{x}=\quad$ Point $1+$ Point $2+$

Line $=$ Letter position of the word $/$ Columns amount

Column $=$ Letter position of the word $-($ Line $*$ Columns Amount)

Thus the position caracter "Line X Column" will activate certain points, as an example:

Letter $_{1 x 1}=1+0+1+1+1+0($ Logic level $)$

results in the activation of the letter "n", as can be visualized in "Fig. 14". 


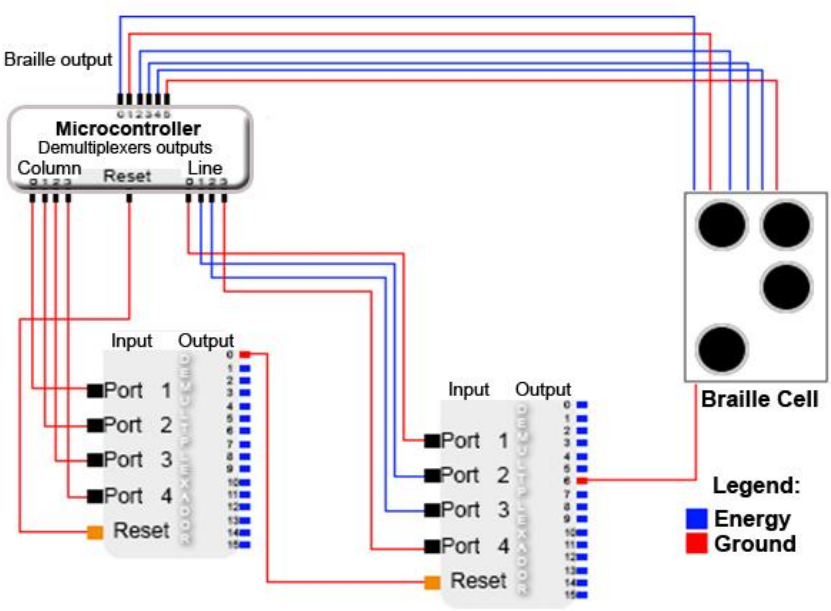

Fig. 14: Connections and activation the Braille cell.

To illustrate the use of the control algorithm and hardware interconnection form, was developed a prototype to validate the techniques, which is described in the following section.

\section{RESULTS AND DISCUSSION}

For purposes of verifying the feasibility of the proposed architecture, were conducted experiments in the laboratory, riding a control circuit in breadboard and fast development board with ATMEGA328P microcontroller. The tests were performed as follows: first establish communication; then the storage capacity of the microcontroller; the use of demultiplexers for selecting an output; test "Print" Braille system; and finally connecting all the components and printed by the solenoid.

In "Fig. 15", it can be seen that the prototype demonstrates multiplexing signals, and performing activation control of the appropriate light emitting diodes (LEDs) and demultiplexer. To test the outputs of Braille characters were used LED's lit up as the character to be "printed". Also the solenoid tests were used to ensure that the operation modes can be sensitive to touch and embossed.

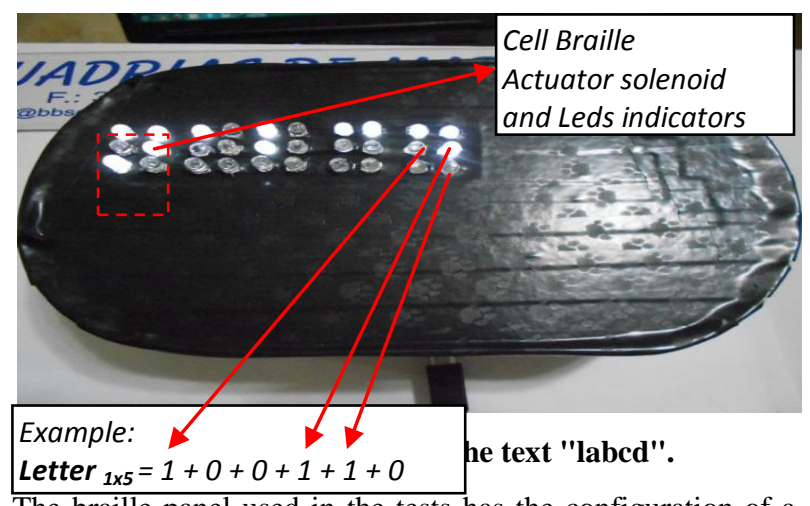

The braille panel used in the tests has the configuration of a line of five columns (1x5), displayed text is "labcd" which can be interpreted in accordance with the firing pattern as in the following example:
Letter $1 \mathrm{x} 1=1+0+1+1+1+0$
Letter $1 \mathrm{x} 2=1+0+0+0+0+0$
Letter $1 \times 3=1+1+0+0+0+0$
Letter $1 \mathrm{x} 4=1+0+0+0+0+0$
Letter $1 \times 5=1+0+0+1+1+0$

For final testing of the control architecture, all components were connected with different configurations of size and the results of these tests in three different braille panel settings can be visualized in the graph of "Fig. 16" below.

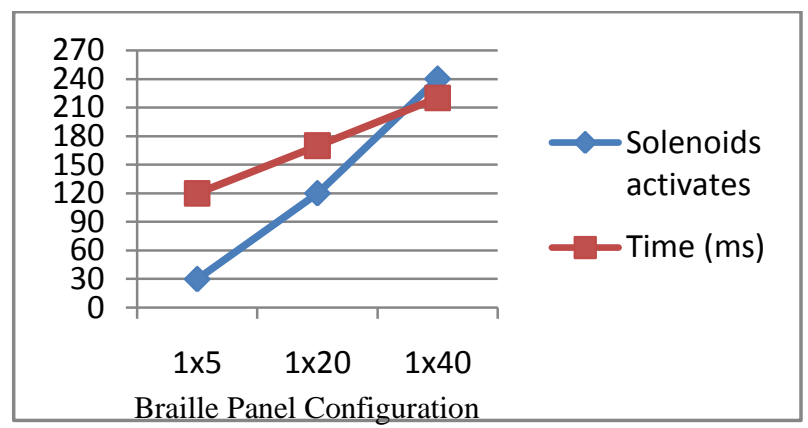

Fig. 16: Graph of solenoid activation tests.

These tests demonstrated that the greater the braille panel, better the performance compared to actuation time due to braille cells actuation technique using demultiplexers. The control system includes software capable of receiving data, translate the text and update the braille panel hardware to the user. The developed control feature allows for a large capacity panel configuration having 512 lines up to 512 columns, resulting in 262,144 Braille characters to be controlled by the signal multiplexing algorithm. This size has an amount of $1,572,864$ actuators (solenoids) and it was estimated an average price of common commercial components and the cost of a small solenoid around \$ 5.00 US dollars.

The control architecture allows the panels configuration with different settings, for example 16 rows by 16 columns (more feasible commercially), totaling 256 characters, therefore, would need 1,536 solenoids, with an average cost of \$ $7,680.00$ all actuators panel. This value can be considered a high cost, but a more usual version with a 16 columns setting for 5 lines obtained a value of $\$ 2,400.00$, which can already be used in environments such as roads, bus stops, airports, universities and other public places. It should also be noted that these values are feasible change, due to be possible a demand or negotiation with industry for this important activity of social inclusion. The following table 2 illustrates some examples of possible configurations with the proposed control architecture.

Table 2. Sample configurations with estimated cost.

\begin{tabular}{|l|l|l|}
\hline Configuration & $\begin{array}{l}\text { Solenoids } \\
\text { amount }\end{array}$ & Estimated cost \\
\hline $16 \times 16-256$ braille cells & 1,536 & $\$ 7,680.00$ \\
\hline $16 \times 8-128$ braille cells & 768 & $\$ 3,840.00$ \\
\hline $16 \times 5-80$ braille cells & 480 & $\$ 2,400.00$ \\
\hline $40 \times 1-40$ braille cells & 240 & $\$ 1,200.00$ \\
\hline
\end{tabular}

Commercial products usually have several configurations, but with the example of a common product with maximum configuration of 40 characters on only one line (for example: Perkins Braille 40 [https://www.enablemart.com/seika-brailledisplay-40- cell]), thus making a brief comparison with a similar configuration gives us the value of $\$ 1,200$ dollars (assuming a price of $\$ 5$ per solenoid), therefore, this prototype 
using solenoids demonstrate the low cost and the various configuration options that the control architecture allows.

In the tests was to validate the proposal as a scalable and parameterizable control according to the need of the user. The features proposed in the methodology, were shipped with the implementation of the algorithm for demultiplexing of signals and the Braille character translation. It was also possible to update the Embedded System in real time is important and satisfying the storage aggregate feature and data recovery in the event of power failure, making the system more reliable.

\section{CONCLUSIONS}

The proposed control architecture shows that it is possible to use demultiplexers for activation control of the characters in Braille cells with a good level of adaptation, reconfiguration and expansion. With the use of solenoid actuators, it can be seen that it is possible to "print" the braille system, with control features agreeing actuators are also a great solution for the tactile representation.

To make it possible that the panel exhibited the Braille system correctly, the study was conducted of it and converted to the source code where the text stored in embedded system is interpreted to be able to read Braille. The upgrade software added to the control architecture offers a preview of how the characters should be on the panel, thus helping those who do not know Braille system also can teach a person with visual impairment to learn the Braille System without having a teacher with very knowledge in the area.

It was verified that the use of the microcontroller the EEPROM memory is limited in space and number of times is recorded, so it would be interesting to add a memory with greater storage and no limits to the amount of recordings. Among the various points raised, the awareness that the job is shows that it is possible to work a technique that seeks expansion in Braille control panels with various settings and with low cost. Thus, to broaden the distribution of information through the use of embedded systems as assistive technologies.

\section{REFERENCES}

[1] OMS, (2012) WHO - World Health Organization. In: <http://www.who.int/blindness/en/> accessed June 2012.

[2] I. B. Constant. (2014) "Vocabulary Braille Basic Concept". In: <http://www.ibc.gov.br>.

[3] Vichitvanichphong, S., Talaei-Khoei, A., Kerr, D., \& Ghapanchi, A. H. (2014). Adoption of Assistive Technologies for Aged Care: A Realist Review of Recent Studies. In System Sciences (HICSS), 2014 47th Hawaii International Conference on (pp. 2706-2715). IEEE.

[4] Jafri, R., Ali, S. A., \& Arabnia, H. R. (2013). Computer vision-based object recognition for the visually impaired using visual tags. In The 2013 International Conference on Image Processing, Computer Vision, and Pattern Recognition (IPCV'13), Las Vegas, Nevada, USA.

[5] Mulloy, A. M., Gevarter, C., Hopkins, M., Sutherland, K. S., \& Ramdoss, S. T. (2014). Assistive Technology for Students with Visual Impairments and Blindness. In Assistive Technologies for People with Diverse Abilities (pp. 113-156). Springer New York.

[6] Ye, M., Li, P., \& Li, Q. (2014, August). VIPReader: A Light News Reader for the Visually Impaired Person. In Proceedings of the 2014 IEEE/WIC/ACM International Joint Conferences on Web Intelligence
(WI) and Intelligent Agent Technologies (IAT)-Volume 02 (pp. 282-287). IEEE Computer Society.

[7] Sonawane, N., Laddad, A., \& Gandhe, S. (2013). Low Cost Braille Embosser Using Speech Hypothesis. In Advances in Computing, Communication, and Control (pp. 510-524). Springer Berlin Heidelberg.

[8] G. B. Zancan; A. S. Roque (2013). "Embedded System for Braille Printing." In: X Congreso Internacional sobre Innovación y Desarrollo Tecnológico, Cuernavaca Morelos, México, IEEE-CS Morelos.

[9] S. Lee; H. S. Hong; W. J. Jeon (2012) "Universal Acess to PDA by Modular I/O Design".

[10] AFB, American Foundation for the Blind. "Braille Displays". In: <http://www.afb.org.>

[11] Siguerdidjane, H., Teodorescu, C. S., Arzandé, A., \& Dugué, F. (2011). Mixed Extended Backstepping-Sliding Modes Control with off line Parameter Optimization used for a class of Solenoid Actuators. IFAC Workshop "Aerospace Guidance, Navigation and Flight Control Systems".

[12] Wolf, W. (2010). High-performance embedded computing: architectures, applications, and methodologies. Morgan Kaufmann.

[13] A. S. Oliveira; A. S. Andrade, F. Souza. "Sistemas Embarcados - Hardware e Firmware na Prática". São Paulo : Érica Ltda., 2006.

[14] IEEE. (1990) "IEEE Stand Glossary of Software Engineering Terminology". Version 610.12-1990, Standards Coordinanting Committee of the IEEE Computer Society, pp. 30, USA.

[15] S. HEATH. "Embedded System Design", 2a ed., Elsevier, 2003.

[16] Rana, M., Sultan, G. M., Mamun Khan, A. A., Hoque, M. N., \& Mitul, A. F. (2013, December). Design and implementation of a GSM based remote home security and appliance control system. In Advances in Electrical Engineering (ICAEE), 2013 International Conference on (pp. 291-295). IEEE.

[17] Wolf, M. (2012). Computers as components: principles of embedded computing system design. Elsevier.

[18] I. B. Gartseev; L. F. Lee; V. N. Krovi. (2011) “A LowCost Real-Time Mobile Robot Platform (ArEduBot) to support Project-Based Learning in Robotics \& Mechatronics", ICRE2011, Moscou, Russia.

[19] Cardesin, J., Garcia-Llera, D., Lopez-Corominas, E., Calleja, A. J., Ribas, J., \& Gacio, D. (2013, December) Low cost intelligent LED driver for public Lighting Smart Grids. In New Concepts in Smart Cities: Fostering Public and Private Alliances (SmartMILE), 2013 International Conference on (pp. 1-6). IEEE.

[20] ATMEL Corporation (Nasdaq: ATMEL) "Microcontrollers manufacturing". In: <http://www.atmel.com/dyn/resourses/.

[21] Tucker, R. S., \& Hinton, K. (2011). Energy consumption and energy density in optical and electronic signal processing. Photonics Journal, IEEE, 3(5), 821-833.

[22] Liu, W., Zhang, M., \& Zhou, X. (2011). The implement of multichannel and adjustable gain A/D sampling system. In Consumer Electronics, Communications and Networks (CECNet), 2011 International Conference on(pp. 1819-1822). IEEE. 